\title{
Produtividade e potencial hídrico foliar do cafeeiro Catuaí, em função da época de irrigação ${ }^{1}$
}

\author{
Antonio C. Silva ${ }^{2}$, Antônio M. da Silva ${ }^{3}$, Gilberto Coelho ${ }^{4}$, Fátima C. Rezende ${ }^{5}$ \& Fabio A. Sato ${ }^{2}$
}

\begin{abstract}
RESUMO
Avaliou-se o efeito de diferentes épocas de irrigação ( $A$ - entre 1 de junho a 30 de setembro; B - entre 1 a 30 de setembro, e, C (Testemunha) - sem irrigação), sobre a produtividade, o rendimento de grãos e o potencial hídrico foliar do cafeeiro (Coffea arabica L.), cultivar Catuaí Vermelho, IAC 44. O experimento foi instalado sobre uma cultura com 18 anos, com espaçamento de 3,5 m entre as linhas de planta por $0,8 \mathrm{~m}$ entre plantas. O potencial hídrico foliar foi monitorado, as 6 h e 12 h, no período de maio a outubro, nos anos de 2003 e 2004. Identificou-se elevada sensibilidade deste indicador fisiológico às épocas de irrigação sendo que a irrigação realizada no período de 01/06 a 30/09 (Tratamento A) mostrou sempre o melhor status hídrico, bem como maior produtividade, com valor médio de $4617 \mathrm{~kg} \mathrm{ha}^{-1}$. Esses resultados ratificam o fato de que a produtividade da cultura guarda relação direta com o seu status hídrico e, sobretudo, o potencial hídrico foliar, que demonstrou ser um indicador fisiológico com poder discriminante, portanto, adequado para ser utilizado como referencial no manejo da irrigação, nas condições estudadas.
\end{abstract}

Palavras-chave: manejo de água, cafeeiro irrigado, indicador fisiológico

\section{Productivity and leaf water potential of the Catuaí coffee crop as a function of irrigation times}

\begin{abstract}
This work evaluated the effect of different periods of irrigation (A - between 06/01 and 09/30; B - between 09/01 and 09/30; C (control) - without irrigation), on productivity, grain yield and leaf water potential of the coffee crop cultivar (Coffea arabica L), cv. Catuaí - Red (IAC 44). The experiment was carried out in 18 years old coffee crop, with $3.5 \mathrm{~m}$ of distance between lines of plants and $0.8 \mathrm{~m}$ between plants. The leaf water potential was evaluated between 0600 and $1200 \mathrm{hrs}$, in the period from May until October for the years 2003 and 2004. The high sensitivity of this physiological indicator to irrigation was identified, and that the irrigation from 06/01 to 09/30 (treatment A) always showed the best leaf water status, as well as the best performance of productivity, with a mean value of $4617 \mathrm{~kg} \mathrm{ha}^{-1}$. The results corroborate with the fact that the productivity of the crop maintained a direct relationship with its leaf water status and especially with the leaf water potential, which showed itself to be a physiological indicator with good discriminating powers, and for this reason, suitable to be utilized as a reference in the irrigation management.
\end{abstract}

Key-words: water management, irrigated coffee, physiology indicator

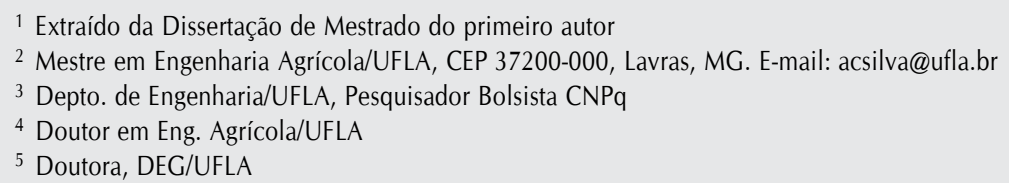




\section{INTRODUÇÃO}

O Brasil é o maior produtor e exportador mundial de café e o segundo mercado consumidor, depois dos Estados Unidos. A irrigação se torna necessária devido aos fenômenos climáticos, como os períodos de veranicos nas fases de maior necessidade de água pela cultura, tornando-se uma técnica de grande utilidade no aumento da produtividade das culturas. Em geral a irrigação é uma prática que, além de incrementar a produtividade, proporciona a obtenção de produto de melhor qualidade e melhor preço no mercado, fatos que também se constatam na cafeicultura irrigada.

Fernandes et al. (2000), usando sistema de irrigação por gotejamento e irrigando o cafeeiro o ano todo, na região de Bonfinópolis, MG, obtiveram produtividade na safra de 1998 igual a 2742 kg ha-1 . Já, Antunes et al. (2000), obtiveram produtividades nas safras de 1999 e 2000 iguais a $2602 \mathrm{~kg} \mathrm{ha}^{-1}$ e $4686 \mathrm{~kg} \mathrm{ha}^{-1}$, respectivamente, ressalta-se que os autores irrigaram o ano todo e fertirrigaram cafeeiros da variedade Catuaí, no município de Rio Preto, MG, região do Campo das Vertentes. Silva et al. (2002) observaram que os tratamentos irrigados apresentaram sensível acréscimo na produtividade acumulada das três primeiras safras.

Parte do sucesso de uma agricultura irrigada está relacionada a um manejo adequado dos recursos naturais solo-água que, interagindo com a atmosfera e a planta, determinam as condições potenciais de máxima produtividade de uma cultura em plenas condições de sanidade e nutrição.

O estudo das relações hídricas no cafeeiro é de particular interesse uma vez que pequenas reduções na disponibilidade da água podem diminuir substancialmente o crescimento, ainda que não se observem murchas nas folhas ou quaisquer outros sinais visíveis do déficit hídrico. Deste modo, a compreensão das relações entre a água e o cafeeiro e suas implicações ecofisiológicas, podem fornecer subsídios ao técnico e ao pesquisador, para tomadas de decisões mais fundamentadas sobre o manejo global da lavoura (Rena \& Maestri, 2000).

Golberg et al. (1988) verificaram que, em condições de campo, a fotossíntese foliar foi pouco afetada até potencial da água foliar da ordem de -1,5 MPa.

Objetivou-se, com este trabalho, avaliar o efeito de diferentes épocas de início de irrigação no cafeeiro, com base no monitoramento e na análise do potencial hídrico foliar e seus reflexos na produção.

\section{MATERIAL E MÉTODOS}

O experimento foi conduzido em uma lavoura de café Coffea arabica L., cultivar Catuaí Vermelho (IAC 44), conduzida com espaçamento de $0,80 \mathrm{~m}$ entre plantas e $3,5 \mathrm{~m}$ entre linhas de plantio, na Fazenda Múquem, Lavras, MG, a uma altitude de $910 \mathrm{~m}$, latitude sul de $21^{\circ} 14^{\prime}$ e longitude oeste de $45^{\circ} 00^{\prime}$.

O solo da área experimental é o Latossolo Vermelho Distróférrico, cujas principais características físico-hídricas estão apresentadas nas Tabelas 1 e 2.
Tabela 1. Granulometria, densidade do solo e densidade de partículas do Latossolo Vermelho Distróférrico

\begin{tabular}{ccccccc}
\hline Camadas & Areia & Silte & Argila & & Ds solo & Dp \\
\cline { 2 - 3 } \cline { 6 - 7 }$(\mathbf{m})$ & & $\left.\mathbf{( g ~ k g - 1}^{-1}\right)$ & & & \multicolumn{2}{c}{$\left(\mathbf{k g ~ d m}^{-3}\right)$} \\
$0-0,20$ & 371,8 & 239,2 & 389,0 & & 1,21 & 2,56 \\
$0,20-0,40$ & 330,9 & 204,0 & 465,1 & & 1,07 & 2,58 \\
$0,40-0,60$ & 330,8 & 176,5 & 492,7 & & 1,03 & 2,59 \\
\hline
\end{tabular}

Tabela 2. Valores de umidade volumétrica $\left(\mathrm{m}^{3} \mathrm{~m}^{-3}\right)$ em função do potencial mátrico $(\mathrm{kPa})$ nas camadas superficiais do Latossolo Vermelho Distroférrico

\begin{tabular}{cccc}
\hline \multirow{2}{*}{ Potencial Matricial } & \multicolumn{3}{c}{ Camada do solo (m) } \\
\cline { 2 - 4 } Solo Sat. & $\mathbf{0 , 0 - 0 , 2 0}$ & $\mathbf{0 , 2 0 - 0 , 4 0}$ & $\mathbf{0 , 4 0 - 0 , 6 0}$ \\
2 & 0,602 & 0,614 & 0,605 \\
6 & 0,481 & 0,447 & 0,461 \\
8 & 0,415 & 0,384 & 0,413 \\
10 & 0,344 & 0,311 & 0,332 \\
33 & 0,305 & 0,297 & 0,310 \\
100 & 0,242 & 0,235 & 0,240 \\
500 & 0,229 & 0,266 & 0,221 \\
1500 & 0,200 & 0,206 & 0,210 \\
& 0,198 & 0,204 & 0,208 \\
\hline
\end{tabular}

O sistema de irrigação constou de uma unidade central de controle, composta por um conjunto motobomba, filtros de areia e de disco, manômetros e linhas de irrigação com tubos flexíveis de polietileno e gotejadores auto-compensantes espaçados $0,3 \mathrm{~m}$ e vazão de 1,6 L h-1 . Os dados climáticos utilizados foram obtidos da estação climatológica da Universidade Federal de Lavras - UFLA, distante 2 km, até o mês de outubro de 2003 e, a partir de então, de uma estação climatológica instalada dentro da área experimental em estudo.

A lâmina de água aplicada entre os meses de junho e setembro foi definida em função da evapotranspiração acumulada entre as irrigações, que foram sempre realizadas nas segundas, quartas e sextas feiras. A transformação da lâmina de irrigação em volume de água foi calculada com base na Eq. 1.

$$
\mathrm{V}=\left[\left(\sum_{\mathrm{I}=1}^{2} \mathrm{ECA} \times \mathrm{K}_{\mathrm{p}} \times \mathrm{K}_{\mathrm{c}}\right)-\mathrm{P}\right] \times \mathrm{A} \times \mathrm{F}
$$

Onde:

V - volume de água a ser aplicado por planta, L;

ECA - evaporação do tanque classe A no período entre irrigações, mm;

$\mathrm{K}_{\mathrm{p}}$ - coeficiente do tanque;

$\mathrm{K}_{\mathrm{c}}$ - coeficiente de cultura para o café, 1,1 (Santatinato, Fernandes \& Fernandes, 1997);

A - área útil entre plantas, 2,8 $\mathrm{m}^{2}$;

$\mathrm{P}$ - precipitação pluvial ocorrida no período, mm e

F - fator de localização, 0,5.

Considerando-se o volume de água a ser aplicado (V) e a vazão do gotejador (q), obteve-se o tempo necessário de irrigação (T), pela Eq. 2.

$$
\mathrm{T}=\frac{\mathrm{V}}{\mathrm{n} \cdot \mathrm{q}}
$$


Onde:

T - tempo necessário de irrigação, h;

V - volume de água a ser aplicado por planta, L;

$\mathrm{n}$ - número de gotejadores por planta;

$\mathrm{q}$ - vazão do gotejador, $\mathrm{L} \mathrm{h}^{-1}$.

O delineamento experimental foi em blocos casualizados com quatro repetições, sendo que cada bloco foi dividido em três parcelas, compostas de uma linha contendo oito plantas, das quais, as seis internas formavam a parcela útil.

As parcelas correspondem às épocas de irrigação, desta forma, a parcela " $A$ " foi irrigada de 1 de junho a 30 de setembro; a parcela " $\mathrm{B}$ ” foi irrigada de 1 a 30 de setembro e a parcela "C" não foi irrigada (Testemunha).

O potencial de água foliar foi avaliado a cada 15 dias, em três plantas, uma em cada parcela, antes do nascer do sol, às 6 h e ao meio-dia, em dias claros, com câmara de pressão do tipo Scholander. Utilizaram-se, em cada medição, três folhas completamente expandidas do terceiro par foliar, a partir do ápice de um ramo plagiotrópico do terço médio da planta (altura mediana), nos períodos das avaliações que se situaram entre maio e setembro nos anos de 2003 e 2004; no intervalo de novembro de 2003 a abril de 2004 não se realizaram avaliações, por se tratar do período de chuvas.

Para medição da umidade relativa (UR) e da temperatura (TF) do ar no dossel, utilizou-se um porômetro de equilíbrio dinâmico, fabricado pela Li-cor, modelo LI 1600; essas variáveis foram utilizadas na determinação do déficit de pressão de vapor (DPV).

A colheita foi realizada manualmente entre os meses junho e agosto, separando-se o café de pano do café de chão, cujo somatório, forneceu a produtividade total. Os dados da produtividade foram submetidos à análise de variância e, quando necessário, ao teste de Scott \& Knott, para comparação das médias; os resultados de produtividade foram expressos $\mathrm{kg} \mathrm{ha}^{-1}$. O rendimento do café beneficiado foi avaliado pelo volume em litros de café da roça necessários para completar uma saca de $60 \mathrm{~kg}$ de café beneficiado.

\section{RESULTADOS E DISCUSSÃO}

\section{Condições ambientais}

Apresentam-se, na Tabela 3, os valores médios de umidade relativa, temperatura foliar e do déficit de pressão de vapor (no momento das avaliações) e valores acumulados da evaporação do tanque classe A e das precipitações pluviais no período de sete dias antes das datas das avaliações. Verifica-se que no ano de 2003 os valores do déficit de pressão variaram de 0,7 a 2,6 kPa, sendo que o maior valor do déficit de pressão de vapor d'água ocorreu na avaliação do dia 23/09/03, sendo explicado pela temperatura elevada, em torno dos $35^{\circ} \mathrm{C}$ e uma umidade relativa bastante baixa em comparação com as outras datas. O menor valor foi constatado no dia 28/08/03, devido a uma chuva de aproximadamente $7 \mathrm{~mm}$ que ocorreu no dia 26/08/03, reduzindo a temperatura e aumentando a umidade relativa; com isto, o déficit de pressão atingiu um valor próximo a $0,7 \mathrm{kPa}$.
Tabela 3. Valores médios de UR $(\%), \operatorname{TF}\left({ }^{\circ} \mathrm{C}\right)$, DPV $(\mathrm{kPa})$ e valores acumulados de ECA $(\mathrm{mm})$ e $\mathrm{P}(\mathrm{mm})$, nos sete dias antes da avaliação

\begin{tabular}{ccccccc}
\hline Ano & Dia/mês & UR (\%) & TF ( $\left.{ }^{\circ} \mathbf{C}\right)$ & DPV (kPa) ECA (mm) & P (mm) \\
& 16 a 25/05 & 63 & 28,2 & 1,4 & 22,98 & - \\
& 13 a 19/06 & 67 & 26,9 & 1,1 & 25,02 & - \\
& 11 a 17/07 & 74 & 23,7 & 0,8 & 21,46 & 14,8 \\
& 25 a 31/07 & 62 & 26 & 1,3 & 30,07 & - \\
2003 a 14/08 & 60 & 29,2 & 1,6 & 26,10 & - \\
& 28 a 28/08 & 74 & 22,0 & 0,7 & 29,66 & 6,2 \\
& 22 a 09/09 & 64,0 & 30,1 & 1,6 & 36,62 & - \\
& 17 a 23/09 & 50,0 & 34,6 & 2,6 & 44,20 & 7,6 \\
01 a 07/10 & 60,0 & 31,0 & 1,8 & 43,41 & - \\
\hline 14 a 20/05 & 84,7 & 25,4 & 0,5 & 20,67 & 28,4 \\
18 a 24/06 & 59,7 & 22,0 & 1,1 & 15,67 & - \\
01 a 08/07 & 67,4 & 22,9 & 0,9 & 16,22 & 1,27 \\
23 a 29/07 & 53,9 & 23,8 & 1,4 & 16,63 & 0,25 \\
06 a 12/08 & 63,4 & 25,0 & 1,2 & 19,19 & - \\
20 a 26/08 & 67,1 & 33,3 & 1,7 & 22,09 & - \\
03 a 09/09 & 49,5 & 33,0 & 2,5 & 19,76 & - \\
\hline
\end{tabular}

No ano de 2004 o déficit de pressão de vapor d’água seguiu o mesmo comportamento do ano de 2003, constatando-se valores entre 0,5 e 2,5 $\mathrm{kPa}$.

\section{Potencial hídrico foliar e produtividade}

Ao longo do período avaliado, o comportamento do potencial hídrico foliar mostrou diferença significativa em nível de $5 \%$, para o fator épocas de irrigação, em 13 das 16 avaliações realizadas às $6 \mathrm{~h}$ e em 12, das 16 avaliações feitas às $12 \mathrm{~h}$.

Apresentam-se, na Tabela 4, os valores médios dos potenciais hídricos foliares às $6 \mathrm{~h}$ e às $12 \mathrm{~h}$, em todas as datas do período de avaliação, sendo que as médias seguidas das mesmas letras não foram diferentes entre si estatisticamente, pelo teste de médias de "Scott \& Knott" e médias não acompanhadas de letras são situações em que não ocorreu diferença estatisticamente significativa entre os tratamentos.

Nas avaliações de 22/05/03 e 20/05/04, quando ainda não se tinha aplicado nenhuma irrigação, os valores médios do potencial hídrico foliar para todos os tratamentos foram bem próximos. Em 07/10/03, quando, se fez a última avaliação do ano, que correspondeu à primeira após o encerramento das irrigações, notou-se, ainda, o efeito significativo da irrigação, porém se observou tendência de redução nos valores médios do potencial hídrico foliar determinado às $6 \mathrm{~h}$, quando comparado com o mês anterior, em que ainda se aplicava o regime de irrigação. Com o início das irrigações em 01/06/2003, ocorreu uma variação nos valores dos potenciais hídricos foliares (PHF) às $6 \mathrm{~h}$. A parcela (A) apresentou valores que variaram de -0,64 a -0,28 MPa; na parcela (B) os valores médios variaram de -0,74 a -0,32 MPa e, na parcela (C) variaram de -1,33 a -0,59 MPa no ano de 2003. Em 2004 os PHF avaliados às $6 \mathrm{~h}$, variaram de $-0,47 \mathrm{a}-0,28 \mathrm{MPa}$ na parcela (A), de -1,04 a -0,39 MPa e -1,31 a -0,49 MPa nas parcelas $(B)$ e $(\mathrm{C})$ respectivamente. 
Tabela 4. Valores médios do potencial hídrico foliar (MPa) do cafeeiro (Coffea arábica), avaliado às $6 \mathrm{~h}$ e às $12 \mathrm{~h}$

\begin{tabular}{|c|c|c|c|c|}
\hline \multirow{2}{*}{ Data } & \multirow{2}{*}{$\begin{array}{c}\text { Horário } \\
\text { (h) }\end{array}$} & \multicolumn{3}{|c|}{ Tratamento - PHF (MPa) } \\
\hline & & A & B & C \\
\hline \multirow{2}{*}{ 22/05/03 } & 6 & $-0,64$ & $-0,61$ & $-0,69$ \\
\hline & 12 & $-1,68$ & $-1,87$ & $-2,42$ \\
\hline \multirow{2}{*}{ 19/06/03 } & 6 & $-0,5 \mathrm{a}$ & $-0,58 \mathrm{a}$ & $-0,96 \mathrm{~b}$ \\
\hline & 12 & $-1,65$ a & $-1,94$ a & $-2,41 b$ \\
\hline \multirow{2}{*}{ 17/07/03 } & 6 & $-0,36$ a & $-0,60 \mathrm{~b}$ & $-1,03 c$ \\
\hline & 12 & $-1,28 \mathrm{a}$ & $-1,98 \mathrm{~b}$ & $-2,65 c$ \\
\hline \multirow{2}{*}{$31 / 07 / 03$} & 6 & $-0,36 \mathrm{a}$ & $-0,69 \mathrm{~b}$ & $-1,29 c$ \\
\hline & 12 & $-1,57 \mathrm{a}$ & $-2,34 \mathrm{~b}$ & $-2,95 c$ \\
\hline \multirow{2}{*}{$14 / 08 / 03$} & 6 & $-0,36 \mathrm{a}$ & $-0,74 b$ & $-1,33 c$ \\
\hline & 12 & $-1,48$ a & $-2,10 \mathrm{~b}$ & $-2,92 b$ \\
\hline \multirow{2}{*}{ 28/08/03 } & 6 & $-0,38$ a & $-0,66 \mathrm{~b}$ & $-0,93 c$ \\
\hline & 12 & $-1,27$ a & $-1,31$ a & $-2,75 b$ \\
\hline \multirow{2}{*}{ 09/09/03 } & 6 & $-0,42 \mathrm{a}$ & $-0,45 \mathrm{a}$ & $-1,10 b$ \\
\hline & 12 & $-1,65$ a & $-2,00 \mathrm{a}$ & $-3,25 b$ \\
\hline \multirow{2}{*}{ 23/09/03 } & 6 & $-0,28$ a & $-0,33$ a & $-0,59 \mathrm{~b}$ \\
\hline & 12 & $-1,88 \mathrm{a}$ & $-2,15 \mathrm{a}$ & $-2,78 b$ \\
\hline \multirow{2}{*}{ 07/10/03 } & 6 & $-0,50 \mathrm{a}$ & $-0,50 \mathrm{a}$ & $-1,20 \mathrm{~b}$ \\
\hline & 12 & $-1,70$ a & $-1,64$ a & $-2,93 b$ \\
\hline \multirow{2}{*}{$20 / 05 / 04$} & 6 & $-0,28$ & $-0,31$ & $-0,39$ \\
\hline & 12 & $-1,40$ & $-1,74$ & $-1,52$ \\
\hline \multirow{2}{*}{$24 / 06 / 04$} & 6 & $-0,32$ a & $-0,39$ a & $-0,60 \mathrm{~b}$ \\
\hline & 12 & $-1,17$ & $-1,48$ & $-1,70$ \\
\hline \multirow{2}{*}{ 08/07/04 } & 6 & $-0,35$ & $-0,40$ & $-0,54$ \\
\hline & 12 & $-1,28$ & $-1,59$ & $-1,83$ \\
\hline \multirow{2}{*}{$29 / 07 / 04$} & 6 & $-0,34 \mathrm{a}$ & $-0,40 \mathrm{a}$ & $-0,55 b$ \\
\hline & 12 & $-1,01 \mathrm{a}$ & $-1,27$ a & $-1,41 \mathrm{~b}$ \\
\hline \multirow{2}{*}{$12 / 08 / 04$} & 6 & $-0,30 \mathrm{a}$ & $-0,81 b$ & $-0,90 \mathrm{~b}$ \\
\hline & 12 & $-1,33$ a & $-1,90 \mathrm{~b}$ & $-1,86 \mathrm{~b}$ \\
\hline \multirow{2}{*}{ 26/08/04 } & 6 & $-0,47 \mathrm{a}$ & $-1,04 \mathrm{~b}$ & $-1,10 b$ \\
\hline & 12 & $-1,69 \mathrm{a}$ & $-2,46 \mathrm{~b}$ & $-2,40 \mathrm{~b}$ \\
\hline \multirow{2}{*}{ 09/09/04 } & 6 & $-0,36$ a & $-0,45$ a & $-1,31 b$ \\
\hline & 12 & $-2,00 \mathrm{a}$ & $-2,32 \mathrm{a}$ & $-2,95 b$ \\
\hline
\end{tabular}

Verificou-se também que em 2003, às $12 \mathrm{~h}$, os valores médios do PHF variaram de $-1,88$ a $-1,27 \mathrm{MPa}$ na parcela (A), de -2,34 a -1,31 MPa na parcela (B) e de -3,25 a $-2,41 \mathrm{MPa}$ na parcela (C). No ano de 2004 variou de $-2,00$ a -1,01 MPa na parcela (A) de -2,46 a -1,27 MPa e -2,95 a -1,41 MPa nas parcelas (B) e (C) respectivamente. Observouse ainda que o menor valor encontrado para o tratamento A foi na avaliação do dia 09/09/04.

Golberg et al. (1988) verificaram que, em condições de campo, a fotossíntese foliar foi pouco afetada até potencial da água foliar da ordem de -1,5 MPa, assim, pode-se afirmar que não houve estresse hídrico às $6 \mathrm{~h}$ em nenhuma das avaliações, em qualquer um dos tratamentos. Os autores afirmaram, ainda, que não houve necessidade de se irrigar o cafeeiro enquanto o teor de água não alcançou a metade da água disponível da capacidade de campo, quando o potencial hídrico foliar mostrou valores próximos de -1,5 MPa, nas horas mais quentes do dia. No potencial hídrico foliar avaliado às $12 \mathrm{~h}$ no tratamento $(\mathrm{A})$, contata-se que $50 \%$ dos valores estão abaixo de $-1,5 \mathrm{MPa}$, porém nunca inferiores a $-2,0 \mathrm{MPa}$.

Embora se tenha constatado uma freqüência elevada de potenciais abaixo de -1,5 MPa, a interação freqüência das irrigações (a cada 2 ou 3 dias) com a elevada capacidade de armazenamento de água do solo ( em torno de $1 \mathrm{~mm} \mathrm{~cm}^{-1}$ de solo), não permitiu caracterizar situação de estresse hídrico, pois sempre houve elevada disponibilidade de água para a planta.

Com relação à parcela (B), constataram-se épocas em que o PHF apresentou valores menores que -2,00 MPa, identificados pela análise estatística como diferenciado da parcela (A) e caracterizando status hídrico inferior. O tratamento não irrigado (C) chegou a um valor mínimo de -3,25 MPa podendo-se inferir que o mesmo foi submetido a um estresse hídrico acentuado sinalizando, portanto, para a necessidade de irrigação.

Quanto à produtividade e ao rendimento, houve diferenças estatisticamente significativas $(\mathrm{P}<0,05)$ entre as épocas de irrigação.

A comparação das médias pelo teste de Scott-Knott, dos resultados de produtividade e rendimento, está apresentada na Tabela 5, na qual se constata que o tratamento (A), que é irrigado de 1/06 a 30/09, apresentou a melhor produtividade, com valor médio de $4617 \mathrm{~kg} \mathrm{ha}^{-1}$, O tratamento que indicou a menor média de produtividade, foi o que recebeu irrigação a partir de 01/09 (B), $1294 \mathrm{~kg} \mathrm{ha}^{-1}$ que não se diferenciou do tratamento não irrigado (C), $1833 \mathrm{~kg} \mathrm{ha}^{-1}$.

Tabela 5. Comparação de médias de produtividade $\left(\mathrm{kg} \mathrm{ha}^{-1}\right)$ e Rendimento (L sc'-1) Safra 2004

\begin{tabular}{ccc}
\hline Épocas de irrigação & Produtividade $\left(\mathbf{k g ~ h a}^{-1}\right)$ & Rendimento $\left(\mathbf{L ~ s c}^{-1}\right)$ \\
A & $4617 \mathrm{a}$ & $595,54 \mathrm{a}$ \\
B & $1294 \mathrm{~b}$ & $487,49 \mathrm{c}$ \\
C & $1833 \mathrm{~b}$ & $539,15 \mathrm{~b}$ \\
\hline
\end{tabular}

Valores seguidos pela mesma letra não se diferem entre si.

A produtividade da parcela A (4617 $\mathrm{kg} \mathrm{ha}^{-1}$ ) foi superior não apenas à produtividade descrita por Antunes et al. (2000) safra 99 (2602 kg ha $\left.{ }^{-1}\right)$ mas também à encontrada por Fernandes et al. (2000) na safra 1998 (2742 $\left.\mathrm{kg} \mathrm{ha}^{-1}\right)$.

O fato da irrigação realizada a partir de 1/06 (tratamento A), propiciar efeitos mais expressivos sobre a produtividade do cafeeiro que outras épocas de inicio da irrigação, também foi constatado por Silva et al. (2002).

Como já discutido, o tratamento (A), apresentou as melhores condições hídricas cujos resultados ratificam o fato de que a produtividade da cultura guarda relação direta com o seu status hídrico e, sobretudo, o potencial hídrico foliar, que demonstrou ser um indicador fisiológico adequado para ser utilizado como referencial no manejo da irrigação, nas condições estudadas.

Quanto ao rendimento, o tratamento A apresentou o valor médio de 595,54 L sc ${ }^{-1}$, ou seja, são necessários 595 litros de café de roça para gerar uma saca de $60 \mathrm{Kg}$ de café beneficiado, e os tratamentos B e C também se diferenciaram entre si, com valores médios 487,49 $\mathrm{L} \mathrm{sc}^{-1}$ e $539,15 \mathrm{~L} \mathrm{sc}^{-1}$, respectivamente, de acordo com a Tabela 5. Verifica-se, então, que o tratamento B foi o que obteve o melhor valor, possivelmente com grãos de café com maior tamanho. 


\section{CONCLUSÕES}

1. Com os resultados obtidos, conclui-se que a irrigação promovida entre junho e setembro propiciou aumento significativo sobre a produtividade do cafeeiro na região de Lavras, Sul de Minas Gerais, correspondendo a mais de 150\% de aumento, em relação ao cafeeiro não irrigado.

2. O potencial hídrico foliar mostrou-se um indicador fisiológico que guarda relação direta com a sua produtividade podendo-se recomendar o seu emprego no manejo da irrigação.

\section{LITERATURA CITADA}

Fernandes, A. L. T.; Santinato, R.; Drumond, L. C. D.; Lessi, R. Irrigação e utilização de granulados de solo na Produção de cafeeiro. In: Simpósio de Pesquisas dos Cafés do Brasil, 9., 2000, Poços de Caldas. Resumos Expandidos... Brasília: Embrapa Café e Minasplan, 2000. v.2, p.957-959.

Golberg, A. D.; Renard, C.; Lannoye, R.; Ledent, J. F. Effects and after-effects of water stress on chlorophyll fluorescence transientsin Coffea canephora Pierre and Coffea arabusta Capot and Aké Assi. Café Cacao Thé, Paris, v.32, n. p.11-16, 1988.

Rena, A. B.; Maestri, M. Relações hídricas no cafeeiro. ITEM, Brasília, n 48, p.64-73, 2000.

Santinato, R.; Fernandes, L. T.; Fernandes, D. R. Irrigação na cultura do café. São Paulo: Arbore, 1997. 146p.

Silva, A. M.; Coelho, G.; Silva, P. A. M.; Coelho, G. S.; Freitas, R. A. Efeito das épocas de irrigação sobre a produtividade do cafeeiro catuai em 4 safras consecutivas. In: Simpósio Brasileiro de Pesquisa em Cafeicultura Irrigada, 2002, Araguari. Anais... Uberlândia: UFU, 2002. p.44-149. 